\title{
A Study of Seventy-Five Courses of Study in Geography
}

\section{Charles M. Reinoehl}

To cite this article: Charles M. Reinoehl (1910) A Study of Seventy\#Five Courses of Study in Geography, Journal of Geography, 8:10, 228-230, DOI: 10.1080/00221341008986233

To link to this article: http://dx.doi.org/10.1080/00221341008986233

曲 Published online: 20 May 2008.

Submit your article to this journal ๘

Џ Article views: 3

Q View related articles $\longleftarrow$ 
various directions from this point, the altitude gauge is advanced along the same guide line to the intersection with the next heavy contour and its elevation adjusted for that point, As before, the surface of the clay is shaped in advance and on the sides so as roughly to conform to the indications of the map; and the same process is repeated until the work of shaping is finished. Corrections for intermediate positions may be carried to any desired refinement which the scale and the accuracy of the map permit.

\section{A STUDY OF SEVENTY-FIVE COÜRSES OF STUDY IN GEOGRAPHY}

By Charles M. Reinoehl

Teachers College, New York City

Age and distribution

20 State Courses, None over three years old, only four older than one year. 55 City Courses, Five older than four years, nearly one-half only one year old or less. All sections of the country represented.

The number and the per cent of the courses of study that omit Geography in the grades. (75 Courses of Study)

Grades,

City,

$\begin{array}{lllllllll}\text { I } & 2 & 3 & 4 & 5 & 6 & 7 & 8 & 9\end{array}$

State,

$\begin{array}{lllllllll}3 \mathrm{I} & 25 & \text { I2 } & 0 & 0 & 0 & 7 & 23 & 5 \mathrm{I}\end{array}$

Total,

$\begin{array}{lllllllll}17 & 15 & 4 & 0 & 0 & 2 & 0 & 8 & \text { I9 }\end{array}$

Per cent,

$\begin{array}{lllllllll}48 & 40 & 16 & 0 & 0 & 2 & 7 & 31 & 70\end{array}$

$\begin{array}{lllllllll}64 & 53 & 2 \mathrm{I} & 0 & 0 & 2.6 & 9.3 & 4 \mathrm{I} & 93\end{array}$

Table showing the number (No.) of Courses of Study that treat World (w) Geography, and the number that treat Home (h) Geography in the Fourth Grade. Of those treating World Geography in this Grade, the number that go through North America only (N.A.).

(X). Grades for which outlines are given.

$\begin{array}{rrrr}r-7, & \text { No. } & \text { w. } & \text { h. } \\ \mathrm{r}-8, & 9 & 7 & 2 \\ & 16 & \text { Io } & 6\end{array}$


I-9,

2-7,

2-8,

3-7,

$3^{-8}$,

3-9,

4-7,

4-6,

4-8,

4-9,

4, 5. 6. 7 ,

$4,5,7,8$

Total,

Indeterminate,

Per cent,

\begin{tabular}{rrr} 
No. & w. & h. \\
3 & 2 & I \\
5 & 3 & I \\
2 & I & I \\
IO & 4 & 3 \\
IO & 4 & 2 \\
2 & & I \\
2 & & 2 \\
2 & & \\
9 & 6 & 3 \\
I & & I \\
I & I & \\
3 & 2 & I \\
\hline & - & - \\
75 & 40 & 24 \\
& - & - \\
& & II
\end{tabular}

Table showing the topics in Geography and their distribution by Grades in the Public Elementary Schools of 55 Cities and 20 States.

Home Geography,

$\begin{array}{rrrrrr}3 & 4 & 5 & 6 & 7 & 8 \\ I 9 & 12 & 2 & & & \\ I 7 & 10 & 3 & \text { I } & \text { I } & 2\end{array}$

City Geography,

County Geography,

State Geography,

$\begin{array}{lllll}I 4 & 4 & 2 & 2 & 2\end{array}$

Earth as a Whole,

United States,

North Ámerica,

South America,

Europe,

Asia,

Africa,

$\begin{array}{llllll}5 & I 2 & 9 & 7 & 5 & 7\end{array}$

$\begin{array}{llllll}9 & 26 & 12 & 3 & 3 & 5\end{array}$

$\begin{array}{lllll}18 & 21 & 17 & 15 & 9\end{array}$

$\begin{array}{llllll}\text { I } & 22 & \text { I9 } & 26 & 12 & \text { I }\end{array}$

$\begin{array}{llllll}\text { I } & 6 & 20 & 20 & 17 & 5\end{array}$

$\begin{array}{lllll}\text { I } & \text { I2 } & \text { I8 } & 24 & 8\end{array}$

I I3 I3 $28 \quad 5$

Australia,

Industrial Geography,

Commercial Geography,

Mathematical Geography,

$\begin{array}{llll}I 3 & 14 & 26 & 6\end{array}$

I3 $13 \quad 26 \quad 6$

$\begin{array}{lllll}2 & 2 & \mathrm{I} & 2 & 9\end{array}$

2 I $5 \quad 7$ 
The order of treatment for continental Geography. In 20 States.

First time over.

$\begin{array}{ccccccc}\text { No. } & \text { N. A. } & \text { S. A. } & \text { Europe. } & \text { Asia. } & \text { Africa. } & \text { Australia. } \\ 8 & \text { I } & 2 & 3 & 4 & 5 & 6 \\ \text { I } & \text { I } & 2 & 4 & 5 & 3 & 6\end{array}$

3 over but once, 8 indeterminate

Second time over.

$\begin{array}{lllllll}7 & \text { I } & 2 & 3 & 4 & 5 & 6 \\ 2 & \text { I } & 6 & 2 & 3 & 4 & 5 \\ \text { I } & 6 & \text { I } & 4 & 5 & 2 & 3 \\ \text { I } & 6 & \text { I } & 2 & 4 & 3 & 5 \\ \text { I } & \text { I } & 3 & 2 & 4 & 5 & 6\end{array}$

I, any order, New York. 7 , indeterminate.

The order of treatment for continental Geography. In 55 Cities.

First time over.

$\begin{array}{lcccccc}\text { No. } & \text { N. A. } & \text { S. A. } & \text { Europe. } & \text { Asia. } & \text { Africa. } & \text { Australia. } \\ 24 & \text { I } & 2 & 3 & 4 & 5 & 6 \\ \text { I } & \text { I } & 2 & 6 & 5 & 3 & 4 \\ \text { I } & \text { I } & 6 & 2 & 3 & 5 & 4 \\ \text { I } & \text { I } & 6 & 2 & 3 & 4 & 5 \\ \text { I } & \text { I } & 5 & 2 & 3 & 4 & 6 \\ \text { I } & 2 & \text { I } & 6 & 3 & 4 & 5\end{array}$

22, indeterminate. 4, part way over.

Second time over.

$\begin{array}{lllllll}32 & \text { I } & 2 & 3 & 4 & 5 & 6 \\ 2 & \text { I } & 2 & 6 & 3 & 4 & 5 \\ 2 & 6 & 5 & \text { I } & 2 & 3 & 4 \\ \text { I } & \text { I } & 2 & 5 & 3 & 4 & 6 \\ \text { I } & \text { I } & 6 & 5 & 2 & 3 & 4 \\ \text { I } & \text { I } & 5 & 6 & 2 & 3 & 4 \\ \text { I } & 4 & 5 & 6 & \text { I } & 2 & 3 \\ \text { I } & 5 & 6 & \text { I } & 2 & 3 & 4\end{array}$

I4, indeterminate. 